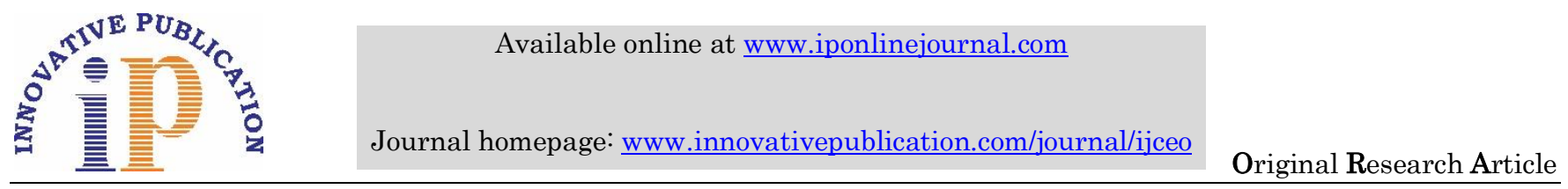

\title{
Study of organisms isolation from acute bacterial conjunctivitis cases
}

\author{
Shadakshari S. Math ${ }^{1}$, Sneh Gautam Rauth ${ }^{2 *}$ \\ ${ }^{1}$ Associate Professor, ${ }^{2} \mathrm{III}{ }^{\mathrm{rd}}$ Year Resident, Dept. of Ophthalmology, D.Y. Patil Hospital Kolhapur, Maharashtra, India
}

\section{Article Info}

Received: $30^{\text {th }}$ March, 2019

Accepted: $5^{\text {th }}$ April, 2019

Published Online: $9^{\text {th }}$ September, 2019

Keywords: Acute conjunctivitis, Gram staining, KOH mounting, Staphylococcus aureus.

\begin{abstract}
Introduction: Conjunctivitis is an inflammation of the outermost layer of the white part of the eye and the inner surface of the eyelid. Conjunctivitis is a global economic burden due to its common occurrence, contagiousness and potentially debilitating morbidities. The bulk of conjunctivitis related costs include consultation, supportive care, drugs prescription, diagnostic tests and productivity loses associated with time away from work or school. Viral etiology was the main reason for most of the outbreaks. Conjunctivitis is one of the most common condition of the eye that affects all ages, social strata and occurs worldwide. Acute conjunctivitis has a symptoms of less than 3 to 4 weeks of duration. Some reports indicate that $50-75 \%$ of acute conjunctivitis are caused due to the bacteria. In adults Staphylococcus, S. pneumoniae and H. influenza are the common pathogens causing bacterial conjunctivitis.

Materials and Methods: Total 70 eyes of 52 patients of diagnosed acute conjunctivitis were examined at our institute from April 2018 to January 2019. This is prospective observational study with evaluation of demographic factors, associated comorbid conditions and finding causative organism i.e. bacteria and /or fungus by gram staining, $\mathrm{KOH}$ mounting and culture sensitivity.

Results: In our study acute conjunctivitis was commonly seen in males than females and in age group of 18-45. It was commonly seen in patients of low socioeconomic status. Predominantly right eye involvement was observed. 16 cases showed positive results for Gram staining. KOH test was negative in all cases. S. aureus was the commonest organism identified on culture and sensitivity. S. aureus and S. pneumonae were equally isolated in patients on chronic steroid use.

Conclusion: Bacterial conjunctivitis is more common i.e. $30.76 \%$ than fungal in our study. No fungal organism was detected in our study. S. aureus, S. pneumonae, H. influenzae and S. epididymis were most common bacterias seen in our study. S. aureus and S. pneumonae were equally isolated in patients on chronic steroid use. We feel that such comprehensive surveys are necessary to assess the specific characteristics of the acute conjunctivitis, which are unique for each region and population.
\end{abstract}

\section{Introduction}

Conjunctivitis is an inflammation of the outermost layer of the white part of the eye and the inner surface of the eyelid. ${ }^{1}$ It makes the eye appear pink or reddish. It may be associated with pain, burning, scratchiness, or itchiness. The affected eye may have increased tears or be "stuck shut" in the morning. Conjunctivitis can affect one or both eyes. ${ }^{2}$ Due to allergies itching is more common. ${ }^{3}$

Viral etiology was the main reason for most of the outbreaks. ${ }^{2,3}$ Even during an outbreak, the number of conjunctivitis cases clinically reported will be less as it is usually benign and self-limiting condition and use of over the counter drugs for the same will decrease the reporting.

Acute conjunctivitis has a symptoms of less than 3 to 4 weeks of duration. Some reports indicate that $50-75 \%$ of acute conjunctivitis are caused due to the bacteria. ${ }^{5}$
The estimated incidence of bacterial conjunctivitis in one study was 135 in 10000. In adults Staphylococcus, Streptococcus pneumoniae and Haemophilus influenza are the common pathogens causing bacterial conjunctivitis. The disease lasts for 7 to 10 days. Clinical features seen in bacterial conjunctivitis include red eye, chemosis and discharge which can be mucopurulent or purulent. The incubation period is 1-7 days. The communicability is $2-7$ days.

\section{Materials and Methods}

\section{Patients}

All patients with acute conjunctivitis presenting to D. Y. Patil Hospital Kolhapur between April 2018 to January 2019.

\footnotetext{
*Corresponding Author: Sneh Gautam Rauth, III ${ }^{\text {rd }}$ Year Resident, Dept. of Ophthalmology, D.Y. Patil Hospital Kolhapur, Maharashtra, India

Email: sneh.rauth@gmail.com

http://doi.org/10.18231/j.ijceo.2019.076
} 
Sample Size: 52 cases

This is prospective observational study with evaluation of demographic factors, associated comorbid conditions and finding causative organism i.e. bacteria and /or fungus by gram staining, $\mathrm{KOH}$ mounting and culture sensitivity. All the cases of acute conjunctivitis attending OPD in D. Y. Patil Hospital from April 2018 to January 2019 and giving written consent for study were included in the study.

\section{Inclusion Criteria}

1. All cases of acute conjunctivitis.

2. All age groups.

3. Both genders

\section{Exclusion Criteria}

1. Patients not willing for giving consent.

2. Ocular trauma.

3. Phlyctenular conjunctivitis

4. Contact lens related red eye

5. Allergic conjunctivitis

\section{Procedure}

A standardized form is filled out on each patient documenting socio-demographic information as well as clinical information including onset of disease, associated comorbid conditions, previous treatment, symptoms, signs. Every patient was examined on the slit lamp biomicroscopy and the ocular signs of conjunctivitis were recorded. Corneal staining done with sterile fluorescein sodium strips to rule out the involvement. In all cases conjunctival swab is obtained on $1^{\text {st }}$ day and sent for Gram staining and $\mathrm{KOH}$ mount to rule out the bacteria and fungi, then patient started on the treatment according to the standard of care. Material obtained from conjunctival swab also inoculated on Blood agar and Mc.Conkey agar. Sensitivity was observed on Muller Hinton agar. In $\mathrm{KOH}$ positive cases culture and sensitivity sent on Sabouraud Dextrose agar.

\section{Results}

Graph 1: Age and sex distribution among the cases.

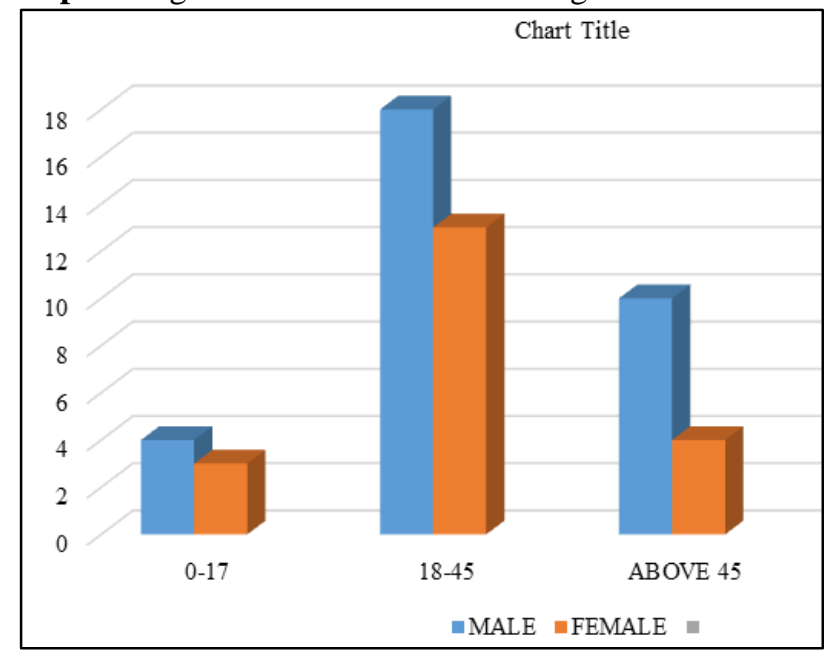

Out of 52 cases high number of cases of acute conjunctivitis were seen in the age group of 18-45. Second age group observed for the cases of acute conjunctivitis is 45 and above. Age group of $0-17$ shows less no of cases. Male preponderance is observed in all age groups.

Graph 2: Socioeconomic distribution

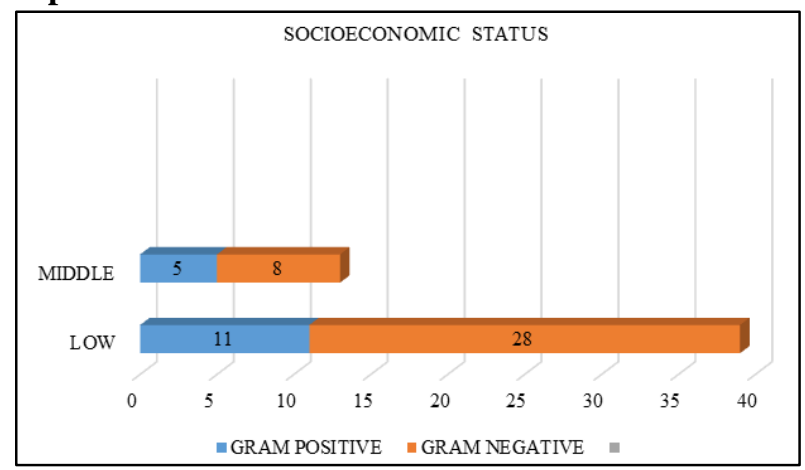

According to our study acute conjunctivitis was observed in low socioeconomic patients as compare to middle socioeconomic status. Total 39 patients were from low socioeconomic status out of which 11 cases are gram positive and 28 cases are gram negative. 13 cases were from middle socioeconomic status out of which 5 are gram positive and 8 are gram negative.

Table 1: Eye involvement

\begin{tabular}{|c|c|c|}
\hline Involved eye & Gram positive & Gram negative \\
\hline LE & 3.08 & 6.92 \\
\hline RE & 7.38 & 16.6 \\
\hline BE & 5.54 & 12.5 \\
\hline
\end{tabular}

This results shoes chi-square $=2.50$ and probability $=0.287$

In 52 cases total 70 eyes were studies. RE involvement was seen predominantly which is 24 . 10 Gram positive cases and 14 Gram negative are seen in RE involvement. Involvement of $\mathrm{BE}$ was seen in 18 cases. 4 cases are Gram positive. Involvement of LE is seen in 10 cases. 2 Gram positive cases and 8 gram negative cases are seen.

Table 2: Percentage of causative bacteria in patients on chronic steroid use

\begin{tabular}{|l|c|}
\hline \multicolumn{1}{|c|}{ Organism } & Percentage \\
\hline Staphylococcus aureus & $33.33 \%$ \\
\hline Streptococcus pnemoniae & $33.33 \%$ \\
\hline Haemophilus influenzae & $16.66 \%$ \\
\hline Staphylococcus epididymis & $16.66 \%$ \\
\hline
\end{tabular}

2 post operative cataract patients did not stopped steroid eye drops but continued it for more than 6 months. 1 patient of chronic uveitis was using steroid eye drops. 2 patients of allergic conjunctivitis were using steroid eye drops. 1 patient of posterior blepharitis used steroid for more than 6 weeks. Total 6 patients were using topical steroid for more than 6 weeks when they presented with acute conjunctivitis. 
Table 3: $\%$ of sign and complications seen in acute conjunctivitis patients in our study

\begin{tabular}{|l|c|c|}
\hline \multicolumn{1}{|c|}{ Signs/complications } & Positive & Negative \\
\hline Lid Edema & $49(94 \%)$ & 3 \\
\hline Matting eyelashes & $13(25 \%)$ & 39 \\
\hline Conjunctival congestion & $52(100 \%)$ & 0 \\
\hline Conjunctival chemosis & $12(23 \%)$ & 40 \\
\hline Petechial Hemorrhage & $50(96 \%)$ & 2 \\
\hline Conjunctival follicles & $47(90 \%)$ & 5 \\
\hline Conjunctival papillae & $20(38 \%)$ & 32 \\
\hline Pseudomembrane & $1(2 \%)$ & 51 \\
\hline Corneal sensation & $51(98 \%)$ & 1 \\
\hline Preauricular lymphadenopathy & $23(44 \%)$ & 29 \\
\hline Complications & & \\
Punctate keratitis & $5(10 \%)$ & 47 \\
Corneal ulcer & $3(6 \%)$ & 49 \\
\hline
\end{tabular}

Table 4: Percentage of causative bacteria

\begin{tabular}{|l|c|}
\hline \multicolumn{1}{|c|}{ Organism } & Percentage \\
\hline Staphylococcus aureus & $66.6 \%$ \\
\hline Streptococcus pnemoniae & $20 \%$ \\
\hline Haemophilus influenzae & $6.66 \%$ \\
\hline Staphylococcus epididymis & $6.66 \%$ \\
\hline
\end{tabular}

\section{Discussion}

The cases were collected between December 2017 to August 2018 with clinical diagnosis of acute conjunctivitis. They were studied and examined at the D.Y. Patil Hospital and Research center Kolhapur. All the cases met the criteria for clinical diagnosis.

In our study out of 52 cases of acute conjunctivitis 28 cases were seen in the age group of 18-45. This age group is more susceptible as this age group is the mainly earning group and active also, they are more exposed to the pathogens. Highest no Gram positive cases are seen in this group. The age group of 45 and above had second highest number of Gram positive cases. The age group of 0-17 shows lowest no of gram positive cases. Close findings were recorded by $\mathrm{Li}$ et al. in a study conducted in a Beijing, China, 2011-2013 shows that people of 18-40 years old are at high risk to be infected with acute conjunctivitis, health education on how to avoid catching this disease should be encouraged among them. ${ }^{6}$

Total 32 male patients were seen in our study which is $61.5 \%$ and 20 female patients were seen which is $38.5 \%$. Similar findings were noted by K. AOKI ET AL, that out of One hundred two patients he studied 62 were men which is $60.7 \%$ and 40 were women which is $39.35^{7}$ The no of male patients is more as they are involved in outdoor activities and physical activities.

In our study out of 52 patients 18 patients had a involvement of both eyes which is $34.6 \%$ and 34 patients had involvement of only one eye which is $65.4 \%$. The involvement of only one eye is seen which can be due to that the patient visit the OPD before the involvement of the other eye. A study conducted by Mini P. Singh, Jagat Ram, ${ }^{1}$ Archit Kumar, Tripti Rungta, Amit Gupta, ${ }^{1}$ Jasmine Khurana, Radha Kanta Ratho in Chandigarh, India in Jan-
March 2018 showed that conjunctivitis was unilateral in 12 patients $(52.2 \%)$ and bilateral in 11 patients $(47.8 \%)^{8}$

In our study out of 52 patients 23 patients had preauricular lymphadenopathy which is $44 \%$ and 47 patients had no preauricular lymphadenopathy which is $56 \%$. A similar finding was noted in a study done by Balasopoulou et al. during March 2012 to June 2012 which shows that out of 231 cases of conjunctivitis preauricular lymphadenopathy was evident in 125 cases which is $54.2 \%{ }^{9}$

According to our study acute conjunctivitis was observed in low socioeconomic patients as compare to middle socioeconomic status. Total 39 patients were from low socioeconomic status which is $75 \%$ and 13 cases were from middle socioeconomic status which is $25 \%$. A study done by Pruthu Thekkur, Mahendra M Reddy, Bijaya Nanda Naik, Subitha L, Sitanshu Sekhar Kar in South India during September - November 2014 also shows that out of 3193 patients 2666 patients were from low socioeconomic status which is $83.5 \%{ }^{10}$

In our study out of 52 patients 15 cases were Gram positive which is $28.8 \%$. A similar study done by $\mathrm{J}$ Clin Pathol in Netherlands in 2009 also found that out of 88 cases 23 cases are Gram positive which is $26.13 \%{ }^{11}$

In our study out of 52 cases 15 case were Gram positive in which 10 cases were Staphylococcus aureus positive which is $66.6 \%$. 3 cases are Streptococcus pneumoniae positive which is $20 \%$ and 1 case showed Haemophilus influenzae positive which was $6.66 \%$. 1 case shows Staphylococcus epididymis positive which was also $6.66 \%$. A study done by J Clin Pathol in Netherlands in 2009 found that out of 88 cases 23 cases were Gram positive which was $26.13 \%$. Out of these 23 cases 12 cases were Staphylococcus aureus positive which was $52.17 \% .6$ cases were Streptococcus pneumoniae positive which was $26 \%$ and 4 case showed Haemophilus influenzae positive which was $17.93 \% .^{12}$

\section{Conclusion}

The purpose of the study was to determine the demographic distribution, associated co-morbid conditions and specific pathogens responsible for acute conjunctivitis.

Acute conjunctivitis is the common problem seen worldwide. It is generally seen in the patients with low socioeconomic status. Prevention of any disease is better than cure which happens to be very true especially regarding to acute conjunctivitis.

Bacterial conjunctivitis is more common i.e. $30.76 \%$ than fungal in our study. No fungal organism was detected in our study. Staphylococcus aureus, Streptococcus pneumonae, Hemophilus influenzae and Staphylococcus epididymis were most common bacteria seen in our study. Staphylococcus aureus and Streptococcus pneumoniae were equally isolated from the acute conjunctivitis patients on chronic steroid use. We feel that such comprehensive surveys are necessary to assess the specific characteristics of the acute conjunctivitis, which are unique for each region and population. 


\section{To Conclude,}

This knowledge is essential, firstly to define the magnitude of the problem in terms of health care costs and the economic burden.

\section{Acknowledgements}

It is with a deep sense of gratitude and reverence that I express my heartfelt thanks to my respected teacher Dr. Shadakshari S. Math, M.S. Ophthalmology, Associate Professor of The Department Of Ophthalmology, Dr. D. Y. Patil Hospital, Kolhapur, who guided me in bringing out this work with his thought provoking ideas and constant encouragement. He was the driving force and constant inspiration throughout the preparation of this study.

I owe my thanks to Dr. Pradeep Dindore, Dr. Sonal Gowaikar and Dr. Supriya Patil whose guidance has been invaluable in completion of this dissertation.

I am also thankful to my colleague Dr. Avani Patil and my junior colleagues Dr. Antariksh Mohta, Dr. Monica Nayyar, Dr. Vedesh Kulkarni and Dr. Hardik Kalaria who have been extremely cooperative throughout my work.

Nothing matches the support of my family, words are not enough to express my gratitude to my parents Dr. Gautam Rauth and Mrs. Aasha Rauth who have been the hidden force to sail me through my journey as a student.

Last but not the least, this acknowledgement is incomplete if I fail in my duty to thank all Patients who have made the study complete.

\section{Source of Funding: None.}

Conflict of Interest: None.

\section{References}

1. Richards A, Guzman-Cottrill JA. "Conjunctivitis". Pediatr Rev 2010;31(5):196-208.doi:10.1542/pir.31-5-196. PMID 20435711

2. Eye Health Information, Pink Eye (Conjunctivitis), "Facts About Pink Eye". National Eye Institute. 2015. Retrieved 8 March 2016.

3. Azari, AA; Barney, NP (23 October 2013). "Conjunctivitis: a systematic review of diagnosis and treatment.". JAMA 2013;310(16):1721-9.doi:10.1001/jama.2013.280318. PMID 24150468.

4. American Academy of Ophthamology. Conjunctivitis;2003;2.

5. Gunnar Hovding; Department of Clinical Medicine, section of Ophthalmology, University of Bergen, Norway; Acute Bactrial Conjunctivitis; Acta Ophthalmol. 2008;86;5-17

6. Li. BMC Infectious Diseases 2018;18:135

7. K. Aoki. Early-Stage Adenoviral Conjunctivitis, Jpn J Ophthalmol 2011;55:11-15

8. Singh MP, Ram J, Kumar A, Rungta T, Gupta A, Khurana J, et al. Molecular epidemiology of circulating human adenovirus types in acute conjunctivitis cases of Chandigarh, North India. Indian J Med Microbiol 2018;36:113-5.

9. Balasopoulou. A molecular epidemiological analysis of adenoviruses from excess conjunctivitis cases. $B M C$ Ophthalmol 2017;17:51;1.

10. Thekkur, Epidemiology of conjunctivitis during an outbreak. Indian J Ophthalmol 2016;64;4;266-71

11. Shenoy KM. Adenoviral Keratoconjunctivitis In Malabar region, Physicians Academy 2012;6;4;57-62.

12. E Tellegen, G ter Riet, J H Sloos, HCPM Van Weert, Diagnosis of conjunctivitis in primary care: comparison of two different culture procedures. J Clin Pathol. 2009;62:939-41.

How to cite this article: Math SS, Rauth SG. Study of organisms isolation from acute bacterial conjunctivitis cases. Indian J Clin Exp Ophthalmol. 2019;5(3):318-21. 